\title{
A determination of propagation constants of symmetric planar waveguide modes in a frequency domain
}

\author{
V.M. Fitio, V.V. Romakh \\ Lviv Polytechnic National University, Department of Photonics, \\ 12, Bandery str. 79013 Lviv, Ukraine \\ Phone/fax: +38(032)-258-25-81; e-mail: polyana@polynet.lviv.ua,vladykv@gmail.com
}

\begin{abstract}
In a new method of finding propagation constants of waveguide modes, the Fourier transform to wave equation is used. We change the differential equation by the integral one. Then we represent the latter equation in a discrete form and get the eigenvalue (square propagation constants)/eigenvector (the discrete Fourier transform of appropriate fields of waveguide modes) problem. Usage of symmetry for symmetric planar waveguides allows to expand opportunities of the new method essentially: to reduce the time of numerical calculation almost triply without loss of analysis accuracy or improve calculation accuracy.
\end{abstract}

Keywords: convolution, Fourier transform, permittivity, propagation constant.

Manuscript received 24.03.14; revised version received 29.07.14; accepted for publication 16.09.14; published online 30.09.14.

\section{Introduction}

To define propagation constants of localized planar waveguide modes, a number of approximate methods are mainly used which are described in detail in $[1,2]$. As usual, these methods for the first time have been developed for analysis of quantum mechanics problems, because one-dimensional stationary Schrödinger equation is almost identical (up to a sign in front of second derivative) to wave equation describing propagation of electromagnetic waves in planar waveguides [3]. One of the most common methods is the WKB method that was proposed for the first time for solution of the stationary Schrödinger equation in 1926. WKB method is also used successfully to analyze gradient waveguides [1], but it is not effective for all profiles of the refractive index.

During last two decades, computer technology has got unprecedented development, moreover, it has high performance, a significant amount of RAM and permanent memory. These powerful computers with appropriate software are accessible to ordinary scientists and engineers. Therefore, numerical methods for solving various problems of physics are now developed, in particular, in electrodynamics.
Solution of the wave equation for planar waveguides by using the numerical method, where differential equation is changed with the corresponding difference one, is proposed $[4,5]$. The latter is represented $N$ times for consistent coordinates $x_{n}$. All these equations together can be written as matrix equation of the eigenvalue (square propagation constants of waveguide modes)/eigenvector (field distribution in discrete form into $x_{n}$ points) problem. By proper choice of numerical process parameters, it can be determined all square propagation constants of waveguide modes among $N$ eigenvalues. This method has an advantage: it enables to define all propagation constants and appropriate fields in one calculation cycle. However, studies of this method have shown that it is characterized by low accuracy.

In $[6,7]$, the new method of finding propagation constants of waveguide modes and appropriate fields in a frequency domain is described. In this method, by the Fourier transform we transfer from the differential wave equation to integral one. In the latter equation, we replace integral with a sum and, as a result, get the problem for eigenvalues (square propagation constants) and eigenvectors (the discrete Fourier transforms of appropriate fields of waveguide modes). The new 
method provides high accuracy subject to conditions of the Whittaker-Shannon sampling theorem [8], and it is characterized by high numerical stability. Also, using this method we define all propagation constants and all the Fourier transforms of corresponding waveguide modes in one cycle of analysis. Detailed reasoning for the new method and its opportunities are given in $[9,10]$.

Besides, it is possible to improve this method significantly and expand its capabilities for symmetric planar waveguides. In this waveguide, permittivity is described by symmetric function with regard to a certain plane, i.e., $\varepsilon(x)=\varepsilon(-x)$. In this case, functions describing the fields are symmetric $(E(-x)=E(x))$ or asymmetric $(E(-x)=-E(x))$. It is clear, we can go from the original matrix equation, where symmetry is not taken into account, to two matrix equations of twice smaller dimension than the original one. It is known that double reduction of matrix dimension reduces the time of numerical calculations by eight times [11]. That is, usage of symmetry of planar waveguide in appropriate matrix equations allows to doubly reduce the matrix dimension (separately for symmetric and asymmetric waveguide modes) and can cause reduction of analysis time approximately three to four times or increase of its accuracy.

\section{One-dimensional wave equations and their Fourier transforms}

An example of a symmetric gradient waveguide is shown in Fig. 1.

If waveguide mode propagates along the axis $z$ and electric field is perpendicular to the plane $x z$ (TE polarization), the appropriate wave equation will look like [12]:

$$
\frac{d^{2} E(x)}{d x^{2}}+\left(\frac{2 \pi}{\lambda}\right)^{2} \varepsilon(x) E(x)=\beta^{2} E(x) .
$$

If in a waveguide, TM polarization wave propagates, the appropriate wave equation with regard to magnetic field can be written as:

$$
\frac{d^{2} H(x)}{d x^{2}}-\frac{\frac{d \varepsilon(x)}{d x}}{\varepsilon(x)} \frac{d H}{d x}+\left(\frac{2 \pi}{\lambda}\right)^{2} \varepsilon(x) H(x)=\beta^{2} H(x) .
$$

The functions $E(x), H(x)$ describe fields in waveguide localized modes, and their first derivatives tend towards zero at $x \rightarrow \pm \infty$. That's why for these functions with their first and second derivatives the Fourier transform exists. One can write appropriate equations for $E(x)$ and its two derivatives:

$$
\begin{aligned}
& \int_{-\infty}^{\infty} E(x) \exp (-i 2 \pi u x) d x=E(u), \\
& \int_{-\infty}^{\infty} \frac{d E(x)}{d x} \exp (-i 2 \pi u x) d x=i 2 \pi u E(u),
\end{aligned}
$$

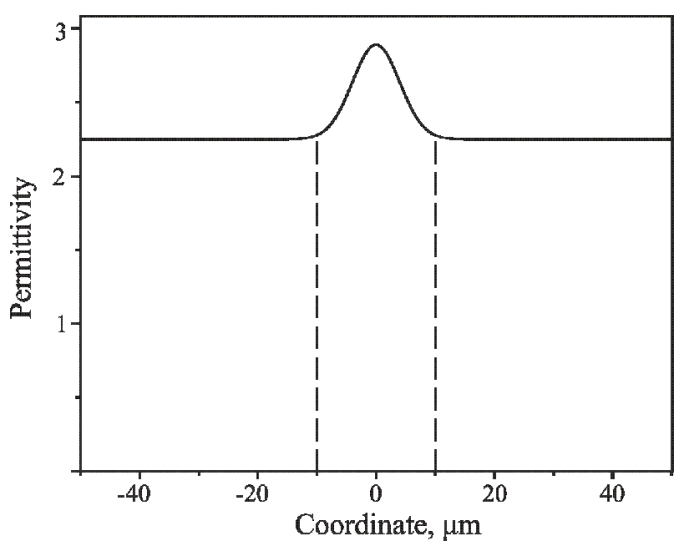

Fig. 1. Dependence of the permittivity on the coordinate according to the function $\varepsilon(x)=2.25+2.79 \exp \left[-\pi(x / 10)^{2}\right]$.

$\int_{-\infty}^{\infty} \frac{d^{2} E(x)}{d x^{2}} \exp (-i 2 \pi u x) d x=-(2 \pi u)^{2} E(u)$.

Besides, for functions for which Fourier transforms exist, i.e., $F\{G(x)\}=G(u), F\{H(x)\}=H(u)$, the next equation is yet right:

$$
F\{G(x) H(x)\}=\int_{-\infty}^{\infty} G(u-v) H(v) d v,
$$

where $F\{\ldots\}$ is the Fourier transform. The expression (6) is named the convolution theorem [8].

Taking Fourier transforms of left and right parts of (1) and (2), as a result we obtain:

$$
\begin{aligned}
& -4 \pi^{2} u^{2} E(u)+\left(\frac{2 \pi}{\lambda}\right)^{2} \int_{-\infty}^{\infty} \varepsilon(u-v) E(v) d v=\beta^{2} E(u) \\
& -4 \pi^{2} u^{2} H(u)-2 i \pi u \int_{-\infty}^{\infty} F\left\{\frac{d \ln \varepsilon(x)}{d x}\right\}_{\mid u \rightarrow u-v} v H(v) d v+
\end{aligned}
$$

$+\left(\frac{2 \pi}{\lambda}\right)^{2} \int_{-\infty}^{\infty} \varepsilon(u-v) H(v) d v=\beta^{2} H(u)$,

where $\varepsilon(u)=\int_{-\infty}^{\infty} \varepsilon(x) \exp (-i 2 \pi u x) d x$.

So, we have transferred from the differential equations (1) and (2) on eigenvalues and eigenfunctions in a coordinate domain to the integral ones (7) and (8) in a frequency domain. In these latter equations, we can replace integrals with sums. For example, if we take (7), resulting in replacement of continuous values $u$ and $v$ on discrete ones we obtain:

$$
\begin{aligned}
& -4 \pi^{2}\left(u_{s}\right)^{2} E\left(u_{s}\right)+\left(\frac{2 \pi}{\lambda}\right)^{2} \times \\
& \times \sum_{k=-N / 2+1}^{N / 2} \varepsilon\left(u_{s}-v_{k}\right) E\left(u_{v}\right) \Delta=\beta^{2} E\left(u_{s}\right)
\end{aligned}
$$


where $\Delta=u_{\max } / N, \quad u_{s}=(s-1 / 2) \Delta, \quad v_{k}=(k-1 / 2) \Delta$, $-N / 2+1 \leq s, k \leq N / 2$.

The value $N$ should be taken enough large and paired. At $N=500$, the value $s$ will be changed from 249 to 250 . One can write the latter equation for a set of discrete frequencies $u_{s}$. Then the set of these equations will be written in the matrix form where $\beta^{2}$ is common to all values of $s$ :

$(\mathbf{P}+\mathbf{U}) \mathbf{E}=\mathbf{B E}=\beta^{2} \mathbf{E}$,

where $\mathbf{P}$ is the diagonal matrix of elements $-4[\pi(s-1 / 2) \Delta]^{2}, \mathbf{U}$ is the square symmetric matrix of elements $\left(\frac{2 \pi}{\lambda}\right)^{2} \varepsilon(s \Delta-k \Delta) \Delta, \mathbf{E}$ is the vector-column of elements $E((s-1 / 2) \Delta)$.

Thus, problem to find the waveguide mode propagation constants leads to the problem on eigenvalues (square propagation constants $\beta^{2}$ ) and eigenvectors (the discrete Fourier transforms $E\left(u_{s}\right)$ in $u_{s}$ points) corresponding to a given value of $\beta$. We can have few eigenvalues and appropriate eigenvectors which are orthogonal. Carrying out the inverse discrete Fourier transform of eigenvector $E\left(u_{s}\right)$ we obtain the field distribution $E(x)$.

If $\varepsilon(x)$ is a symmetry function, the elements $b_{i, j}$ of matrix B satisfy following symmetry:

$b_{i, j}=b_{j, i}, b_{i, j}=b_{N+1-i, N+1-j}$,

where subscripts $i$ and $j$ change from 1 to $N$, and are related with subscripts $s$ and $k$ in the following way: $i=s+N / 2, j=k+N / 2$. So, we transfer from $E\left(u_{s}\right)$ to $E\left(u_{i}\right)$.

First consider the case when $E(x)$ is a symmetric function, $E\left(u_{s}\right)$ will be also symmetric with regard to $u_{s}$. To obtain matrix equation of the type (9) with two-fold smaller dimension, add term by term the first and $N$-th lines of the matrix equation (10), then the second and $(N-1)$-th lines and so on. Moreover, we introduce the notations:

$$
\begin{aligned}
& E^{+}\left(u_{1}\right)=E\left(u_{1}\right)+E\left(u_{N}\right), \\
& E^{+}\left(u_{2}\right)=E\left(u_{2}\right)+E\left(u_{N-1}\right) \text {, } \\
& E^{+}\left(u_{i}\right)=E\left(u_{i}\right)+E\left(u_{N-i+1}\right), \\
& E^{+}\left(u_{N / 2}\right)=E\left(u_{N / 2}\right)+E\left(u_{N / 2+1}\right) \text {. }
\end{aligned}
$$

In case of symmetric field distribution of waveguide mode, we calculate elements of matrix $\mathbf{B}^{+}$by elements of matrix $\mathbf{B}$, taking into account expressions (11) and (12):

$b_{i, j}^{+}=b_{i, j}+b_{N-i+1, j}$.
We get the matrix equation for the eigenvalue/eigenvector problem, but only for these propagation constants that correspond to symmetric functions $E(u)$ and, therefore, to $E(x)$ :

$\mathbf{B}^{+} \mathbf{E}^{+}=\beta^{2} \mathbf{E}^{+}$.

For asymmetric field distribution we carry out analysis in a like manner. As the function $E(x)$ is asymmetric, $E\left(u_{s}\right)$ is also asymmetric function with respect to $u_{s}$. For obtaining matrix equation like (9) but of twice smaller dimension, subtract $N$-th line from the first one of the matrix equation (10), then $(N-1)$-th line from the second and so on. In addition, we introduce the notations again:

$$
\begin{aligned}
& E^{-}\left(u_{1}\right)=E\left(u_{1}\right)-E\left(u_{N}\right), \\
& E^{-}\left(u_{2}\right)=E\left(u_{2}\right)-E\left(u_{N-1}\right) \text {, } \\
& E^{-}\left(u_{i}\right)=E\left(u_{i}\right)-E\left(u_{N-i+1}\right), \\
& E^{-}\left(u_{N / 2}\right)=E\left(u_{N / 2}\right)-E\left(u_{N / 2+1}\right) \text {. }
\end{aligned}
$$

For asymmetric field distribution elements of matrix $\mathbf{B}^{-}$are respectively:

$b_{i, j}^{-}=b_{i, j}-b_{N-i+1, j}$.

In this case, the matrix equation for those propagation constants that correspond to asymmetric functions $E(u)$ and $E(x)$ has the form:

$\mathbf{B}^{-} \mathbf{E}^{-}=\beta^{2} \mathbf{E}^{-}$.

Subscripts $i$ and $j$ change from 1 to $N / 2$ in the equations (14) and (17).

\section{Numerical analysis}

A numerical analysis was carried out for the waveguide which permittivity was described by:

$$
\varepsilon(x)=\left\{\begin{array}{l}
\varepsilon_{0}+\left(\varepsilon_{1}-\varepsilon_{0}\right)\left[1-(2 x / d)^{2}\right], \text { if }|x|<d / 2, \\
\varepsilon_{0}, \text { if }|x|>d / 2,
\end{array}\right.
$$

where $\varepsilon_{0}=2.25, \quad \varepsilon_{1}=2.89, \quad d=20 \mu \mathrm{m}, \quad \lambda=1 \mu \mathrm{m}$. Numerical process parameters are as follows: $N=3000$, $u_{\max }=30 \mu \mathrm{m}^{-1}$. For waveguide (quadratic profile) of permittivity distribution described by equation (18), 25 propagation constants were found, among which 13 values correspond to symmetric field distribution, and 12 ones - to asymmetric. In particular, the value $\beta_{0}=10.657860 \mu \mathrm{m}^{-1}$ is a constant at frequency change $u_{\max }=5 \ldots 150 \mu \mathrm{m}^{-1}$, and the value $\beta_{24}=9.462809 \mu \mathrm{m}^{-1}$ is constant for frequency range $u_{\max }=10 \ldots 80 \mu \mathrm{m}^{-1}$. We have got exactly the same results when: a) we searched propagation constants by (10), where the size of matrix 


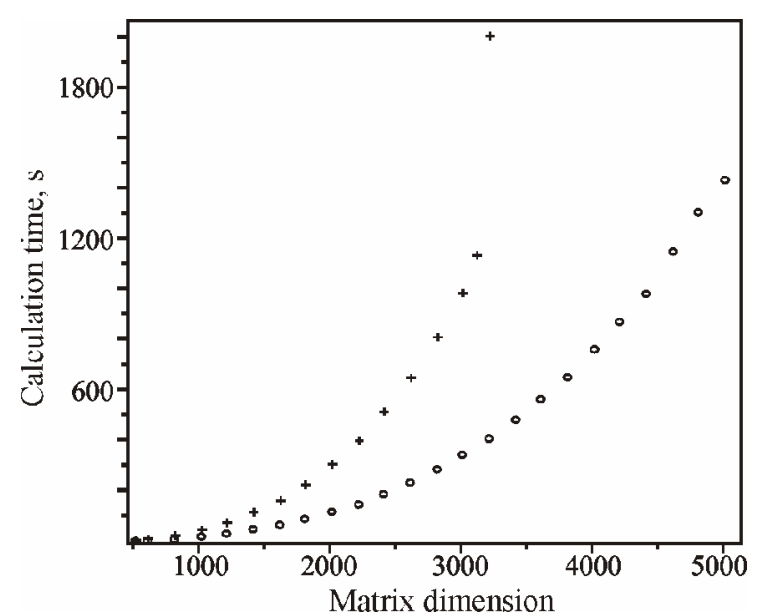

Fig. 2. Dependence of the calculation time on the matrix dimension.

B is $N \times N ;$ b) we used a symmetry according to expressions (14) and (17), where the size of corresponding matrices $\mathbf{B}^{ \pm}$is $N / 2 \times N / 2$. However, in the second case, the time of analysis is less about 2.9 times at $N=3000$.

In Fig. 2, the dependences of calculation time on matrix dimension $N$ without symmetry (cross) and its consideration (circle) are shown. It is seen that when we take into account symmetry of permittivity distribution the dependence of time on matrix dimension $N$ is more sloping than without it. Therefore, usage of symmetry allows to use the matrix dimension larger than 5000. If it isn't used, calculation time increases dramatically at $N=3200$, and its value is about 2000 s, i.e., more than half an hour. So, further increase of the $N$ value is too time-consuming.

\section{Conclusions}

Applying this approach allows to carry out analysis of planar waveguides that have much higher propagation constants of waveguide modes than in the example shown above. Apparently, calculation time in first and second cases depends on PC, software and programmer skills. However, it is evident that main results regarding usage of symmetry are unchanged: significant reduction in calculation time and/or new opportunities of the method proposed.

\section{References}

1. A.W. Snyder and J.D. Love, Optical Waveguide Theory. Chapman and Hall, London, New York, 1983.

2. H.-G. Unger, Planar Optical Waveguides and Fibres. Clarendon Press, Oxford, 1977.

3. I.O. Vakarchuk, Quantum Mechanics. Ivan Franko National University of Lviv, Lviv, 2004.

4. A.G. Rganov and S.E. Grigas, Defining the parameters of multilayer waveguide modes of dielectric waveguides // Numerical Methods and Programming, 10(1), p. 258-262 (2009).

5. A.G. Rganov and S.E. Grigas, Numerical algorithm for waveguide and leaky modes determination in multilayer optical waveguides // Technical Physics, 55(11), p. 1614-1618 (2010).

6. V.M. Fitio, V.V. Romakh, Y.V. Bobitski, Localized modes of the gradient planar waveguides. Analogies in the quantum mechanics // Electronics and Information Technologies, 1, p. 134-141 (2011).

7. V.M. Fitio, V.V. Romakh, Y.V. Bobitski, A solution of the wave equation for planar gradient waveguides in a frequency domain // 6th Intern. Conf. on Adv. Optoelectronics \& Lasers, Sudak, Ukraine, p. 240-242 (2013).

8. J.W. Goodman, Introduction to Fourier Optics. McGraw-Hill Book Company, San Francisco, 1968.

9. V.M. Fitio, V.V. Romakh, Y.V. Bobitski, Numerical method for analysis of waveguide modes in planar gradient waveguides // Mater. Sci. (accepted 23.01.2014).

10. V.M. Fitio, V.V. Romakh, Ya.V. Bobitski, The Fourier transform application to search for localized modes of gradient planar waveguides // $J$. Radiophys. and Electronics, 18(4), p. 21-26 (2013).

11. L. Li, Formulation and comparison of two recursive matrix algorithms for modeling layered diffraction gratings // J. Opt. Soc. Am. A, 13(5), p. 1024-1035 (1996).

12. A. Yariv and P. Yeh, Optical Waves in Crystals: Propagation and Control of Laser Radiation. John Wiley \& Sons, New York, 1984. 